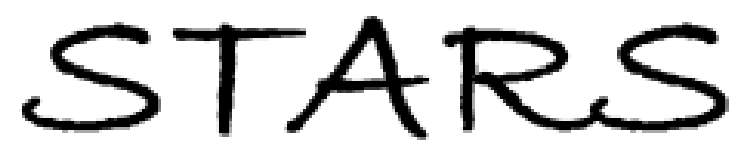

University of Central Florida

STARS

$1-1-1996$

\title{
Area-Selective Disordering Of Multiple Quantum Well Structures And Its Applications To All-Optical Devices
}

Ayman M. Kan'an

University of Central Florida

Patrick LiKamWa

University of Central Florida

Mitra Dutta

Jagadeesh Pamulapati

Find similar works at: https://stars.library.ucf.edu/facultybib1990

University of Central Florida Libraries http://library.ucf.edu

This Article is brought to you for free and open access by the Faculty Bibliography at STARS. It has been accepted for inclusion in Faculty Bibliography 1990s by an authorized administrator of STARS. For more information, please contactSTARS@ucf.edu.

\section{Recommended Citation}

Kan'an, Ayman M.; LiKamWa, Patrick; Dutta, Mitra; and Pamulapati, Jagadeesh, "Area-Selective Disordering Of Multiple Quantum Well Structures And Its Applications To All-Optical Devices" (1996).

Faculty Bibliography 1990s. 1654.

https://stars.library.ucf.edu/facultybib1990/1654

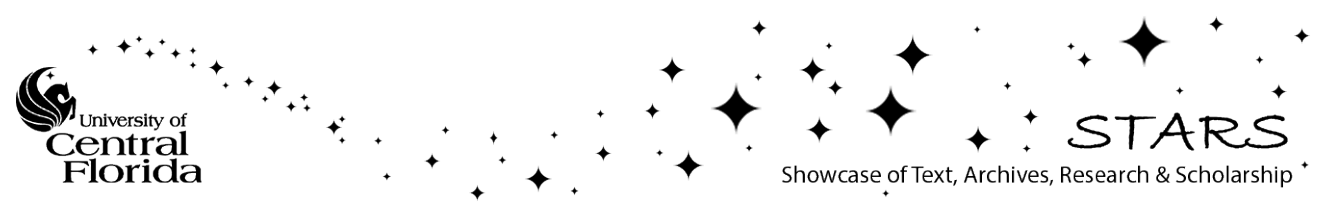




\section{Area-selective disordering of multiple quantum well structures and its applications to all-optical devices}

Cite as: Journal of Applied Physics 80, 3179 (1996); https://doi.org/10.1063/1.363257

Submitted: 03 May 1996. Accepted: 12 June 1996. Published Online: 04 June 1998

Ayman M. Kan'an, Patrick LiKamWa, Mitra-Dutta, and Jagadeesh Pamulapati

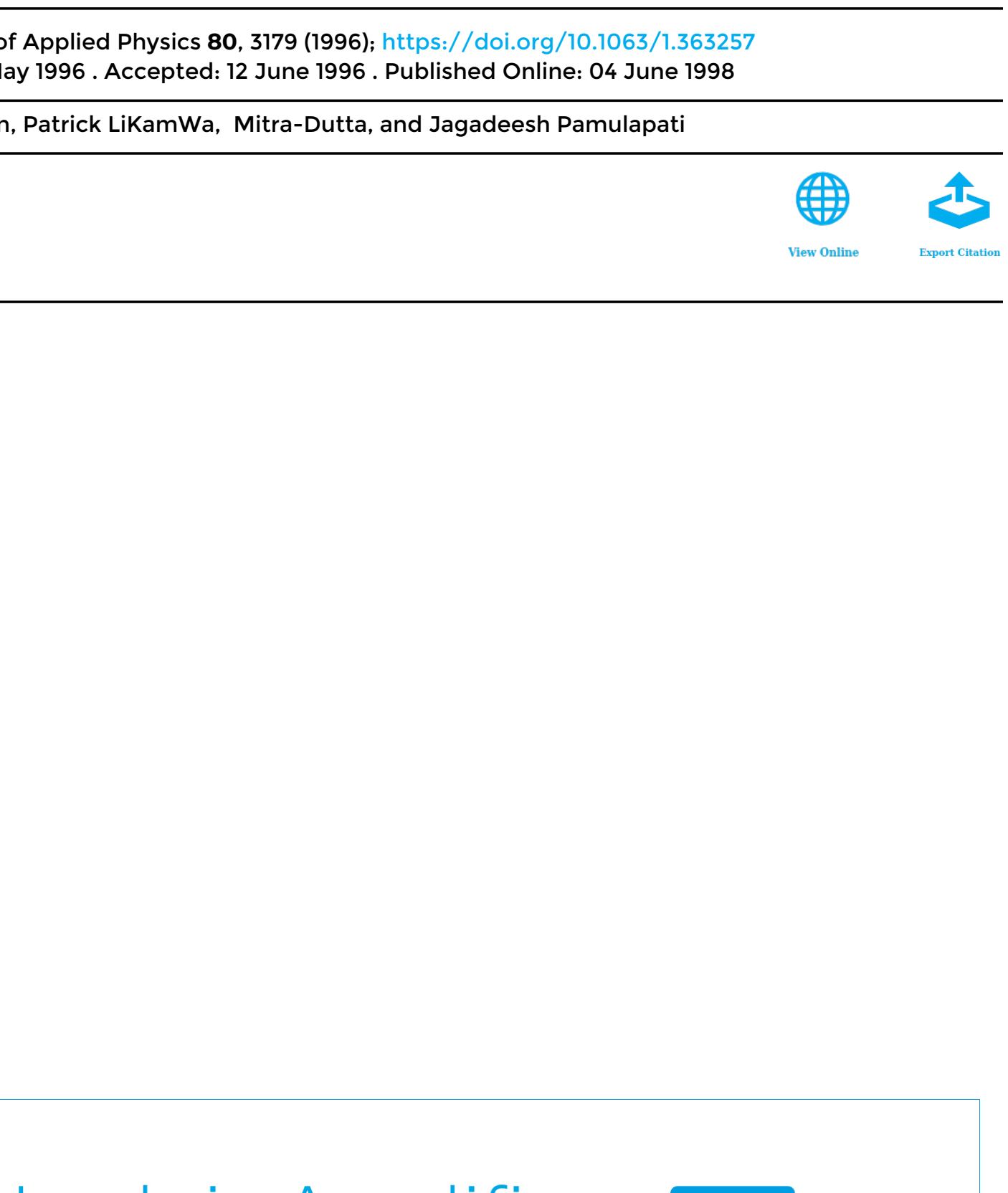

Lock-in Amplifiers

... and more, from DC to $600 \mathrm{MHz}$ Watch 


\title{
Area-selective disordering of multiple quantum well structures and its applications to all-optical devices
}

\author{
Ayman M. Kan'an and Patrick LiKamWa $a^{\text {a),b) }}$ \\ Center for Research and Education in Optics and Lasers (CREOL), University of Central Florida, \\ PO Box 162 700, 4000 Central Florida Boulevard, Orlando, Florida 32816-2700 \\ Mitra-Dutta and Jagadeesh Pamulapati \\ U.S. Army Research Laboratory, 2800 Powder Mill Road, Adelphi, Maryland 20783-1145
}

(Received 3 May 1996; accepted for publication 12 June 1996)

\begin{abstract}
A technique of impurity-free vacancy-induced disordering of GaAs/AlGaAs multiquantum wells (MQW) that is area selective, very reliable, and highly reproducible, has been developed. The localized compositional disordering is induced by rapid thermal annealing of the sample after it has been coated with a thin film of "spin-on" glass and prebaked at $400{ }^{\circ} \mathrm{C}$ in a high purity nitrogen:oxygen (78:22) atmosphere. In order to self-consistently determine the diffusion coefficient of the $\mathrm{Al}$ and $\mathrm{Ga}$ atoms, the photoluminescence peak is fitted to the $n=1$ electron to heavy hole transition that corresponds to an error function potential profile caused by the diffusion. The process has been used to integrate two optical devices on a MQW structure. One is a nonlinear directional coupler all-optical switch, and the other is an integrated Mach-Zehnder all-optical modulator. The switching characteristics of the devices were measured using the conventional pump-probe measurement technique. (C) 1996 American Institute of Physics. [S0021-8979(96)06018-5]
\end{abstract}

\section{INTRODUCTION}

Post-growth band gap engineering of multiquantum well structures $(\mathrm{MQW})$ is an essential key requirement for the fabrication of integrated optoelectronic devices and low-loss waveguides on a single wafer. ${ }^{1}$ Many devices, such as optical modulators and monolithic band gap tuned lasers, rely heavily on quantum confinement for their operation and offer improved performance over their bulk counterparts. Other components, such as interconnects and strip-loaded waveguides, need to operate at optical frequencies that are nonresonant with the band gap frequency of the semiconductor so that the waveguide is effectively transparent at the operating wavelength. To achieve monolithic integration of several optoelectronic devices on a single MQW wafer, one should be able to define materials with different optoelectronic properties (absorption coefficient, material resistivity, and refractive index) at different locations. Although MQWs with different compositions and thicknesses can be realized during growth, ${ }^{2}$ this method of integrating optoelectronic devices requires extensive growth capabilities and the growth conditions are very critical for the realization of high quality structures and achieving reproducible results.

A number of techniques have already been developed and applied to achieve the controlled post-growth intermixing of MQWs. Such techniques are laser-induced disordering, ${ }^{3,4}$ ion-implantation-enhanced interdiffusion, ${ }^{5,6}$ impurity-induced disordering (IID), ${ }^{7}$ and impurity-free vacancy diffusion (IFVD).$^{8-13}$ In the case of IID, impurities induce the disordering process through generating free carriers that increase the number of vacancies at the annealing temperature. The diffusion coefficient of $\mathrm{Al}$ in an undoped

\footnotetext{
${ }^{a}$ Electronic mail: likamwa@creol.ucf.edu

${ }^{\text {b) }}$ Also with the Dept. of Electrical and Computer Engineering, University of Central Florida.
}

AlAs-GaAs heterostructures is of the order of $10^{-19} \mathrm{~cm}^{2} \mathrm{~s}^{-1}$ at $850{ }^{\circ} \mathrm{C}^{14}$ However the diffusion coefficient is dramatically enhanced when impurities such as $\mathrm{Zn}, \mathrm{Si}$, or $\mathrm{Be}$ are introduced into the heterostructure. A serious problem associated with the use of $\mathrm{Zn}$ and $\mathrm{Si}$ as the impurities in IID is the free carrier absorption. On the other hand using electrically neutral dopants (F and B) as IID species results in other problems like generating traps associated with these species and residual damage from implantation.

Among all methods of MQW intermixing, IFVD is considered to be the most promising method because it retains high crystal quality and low optical propagation losses due to its low free carrier concentration. There are two problems that have been associated with IFVD, namely in area selectivity and in reproducibility.

In the present article, we describe a simple, inexpensive, yet very reliable method of achieving post-growth IFVD intermixing of GaAs/AlGaAs MQWs. This technique is affordable, area selective, and highly reproducible. Room temperature photoluminescence (PL) characterization indicates a $40 \mathrm{~nm}$ possible blue shift in the peak emission wavelength as a result of the disordering process. We used this process to realize two devices: One is an all-optical switch that has a nonlinear mode-beating section coupled with low-loss branching waveguides. The other is an integrated MachZehnder optical modulator that has one nonintermixed arm while the other arm and the rest of the structure are made of disordered MQW structure. Pump-probe measurements were done on the devices to study the switching characteristics of these integrated devices. The organization of this article is as follows: The experimental procedure and results are described in Sec. II. A simple theory is described in Sec. III. Applications of the new process in the fabrication of integrated waveguide switches are given in Sec. IV. Finally, Sec. $\mathrm{V}$ is devoted to a brief discussion. 


\section{EXPERIMENTAL PROCEDURE}

The MQW waveguide structure used in this work consists of 38 pairs of GaAs and $\mathrm{Al}_{0.3} \mathrm{Ga}_{0.7} \mathrm{As}$ layers each $80 \AA$ thick, and confined on each side by $\mathrm{Al}_{0.3} \mathrm{Ga}_{0.7} \mathrm{As}$ cladding layers that are 0.5 and $2.5 \mu \mathrm{m}$ thick on the top and the bottom, respectively. The undoped layers were grown lattice matched by molecular beam epitaxy on an undoped GaAs substrate. In order to achieve controlled intermixing between the GaAs quantum wells and the AlGaAs barriers, the surface of the sample was coated with a thin film of "spin-onglass" which is a commercially available solution of glass $\left(\mathrm{SiO}_{x}\right)$ forming compound. The thin film was deposited by spinning the liquid on the sample surface at a speed of 3000 rpm for $30 \mathrm{~s}$, this resulted in a film thickness of $230 \mathrm{~nm}$. The film was then cured at $400{ }^{\circ} \mathrm{C}$ for $30 \mathrm{~min}$ in a constant flow of ultrahigh purity air (78\% nitrogen and $22 \%$ oxygen). Localized compositional disordering was then induced by photolithographic definition and removal of the $\mathrm{SiO}_{x}$ film in selected regions followed by rapid thermal annealing. The rapid heating of the sample was carried out in a flowing nitrogen atmosphere in an AG Associates Heatpulse 210 rapid thermal annealer. The sample was placed face down on a mechanical grade undoped GaAs substrate to protect the face of the sample and to provide overpressure of As so as to minimize its desorption. The silicon oxide cap at the surface promotes out diffusion of $\mathrm{Ga}$ atoms into the cap layer, thus generating group III vacancies at the surface of the sample. The intermixing is caused by the inter diffusion of the these vacancies with $\mathrm{Ga}$ and $\mathrm{Al}$ atoms, with a subsequent diffusion of the vacancies into the MQW region and the ensuing disordering of the quantum wells. There have been previous reports on the use of $\mathrm{SiO}_{2}$ to enhance the intermixing of group III-V multiquantum well structures. ${ }^{11,12,15}$ Rapid thermal annealing of a sample that is encapsulated by an $\mathrm{SiO}_{x}$ cap layer deposited on the surface by either chemical vapor deposition or electron beam evaporation results in a significant blue shift of the band edge. The magnitude of the blue shift is a function of the annealing time and temperature. The main difference between this work and the previously reported work is the simplicity of the current technique that is used to generate the vacancies and enhance the impurity free vacancy diffusion in the heterostructure.

In order to characterize our method of IFVD, different samples measuring $3 \times 4 \mathrm{~mm}^{2}$ of the MQW structure described above were first cleaned with solvents, and then coated with spin-on glass and cured at $400{ }^{\circ} \mathrm{C}$ for $30 \mathrm{~min}$ in a nitrogen/oxygen atmosphere. Conventional photolithography and photoresist masking was then used to etch off the $\mathrm{SiO}_{x}$ layer from the surface of one half of each sample by immersing them in a buffered oxide etchant (BOE) (1:9 $\mathrm{HF}: \mathrm{H}_{2} \mathrm{O}$ ) for $30 \mathrm{~s}$. The samples were then annealed separately for $20 \mathrm{~s}$ at temperatures of $960,970,980$, and $1000{ }^{\circ} \mathrm{C}$, respectively. The degree of disordering was measured by comparing the room temperature $\mathrm{PL}$ of the $\mathrm{SiO}_{x}$-capped region, the uncapped region, and an as-grown sample. The samples were excited by a focused $15 \mathrm{~mW} \mathrm{He}-\mathrm{Ne}$ laser beam $(632.8 \mathrm{~nm})$, and the PL was collected by a lens onto a monochromator connected to an optical multichannel analyzer. As the $\mathrm{Al}$ atoms diffuse into the quantum well region,

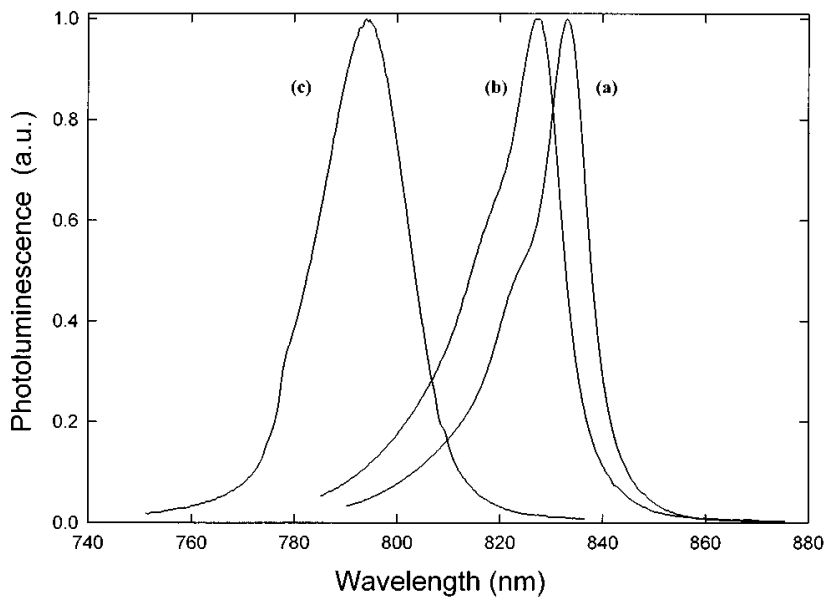

FIG. 1. Room temperature photoluminescence spectra of the MQW samples: (a) from the as-grown sample; (b) from the annealed sample with no film on its surface, and (c) from the annealed $\mathrm{SiO}_{x}$-capped sample.

the abruptness of the interfaces is destroyed and the subband energies move apart, resulting in an increase in the $n=1$ electron to heavy-hole transition energy. Figure 1 shows the measured room temperature photoluminescence of (a) an asgrown sample, (b) an annealed sample with no film on its surface, and (c) an annealed silicon oxide film coated sample. The thermal annealing was carried out at $980{ }^{\circ} \mathrm{C}$ for $20 \mathrm{~s}$. The heavy hole (hh) and light hole (lh) exciton transitions are clearly resolved for the as-grown and the uncapped sample. The measurement indicates that the MQW intermixing using this method results in a difference of $37 \mathrm{~nm}$ between the blue shift of the effective band edge of the coated and the uncoated samples.

\section{THEORETICAL MODELING}

The Al-Ga interdiffusion across the heterointerfaces has been modeled using an error function profile. ${ }^{14}$ For a quantum well centered at $z=0$, the $\mathrm{Al}$ composition varies across the well as

$$
x(z)=x_{0}\left[1+\frac{1}{2} \operatorname{erf}\left(\frac{z-W / 2}{2 \sqrt{D t}}\right)-\frac{1}{2} \operatorname{erf}\left(\frac{z+W / 2}{2 \sqrt{D t}}\right)\right],
$$

where $x_{0}$ is the initial $\mathrm{Al}$ concentration in the region surrounding the $\mathrm{QW}, W$ is the initial well width, $D$ is the diffusion coefficient assuming isotropic $\mathrm{Al}-\mathrm{Ga}$ interdiffusion, $t$ is the annealing time, and erf() is the error function. To find the diffusion coefficient at a given annealing time and temperature, we assume that the quantum well closest to the surface contributes to the part of the PL at its peak wavelength. We also assume that there is no coupling between the neighboring quantum wells. This assumption is valid because the barrier separating the wells is thick enough to prevent any noticeable interaction between the wave functions of the eigenstates inside the QWs. Thus, we can safely assume that the calculated $e l$-hh1 transition energy of a single well corresponds to that of our MQW structure. In the calculations of the electron and heavy-hole subband energies, the following parameters were used: the effective masses of 


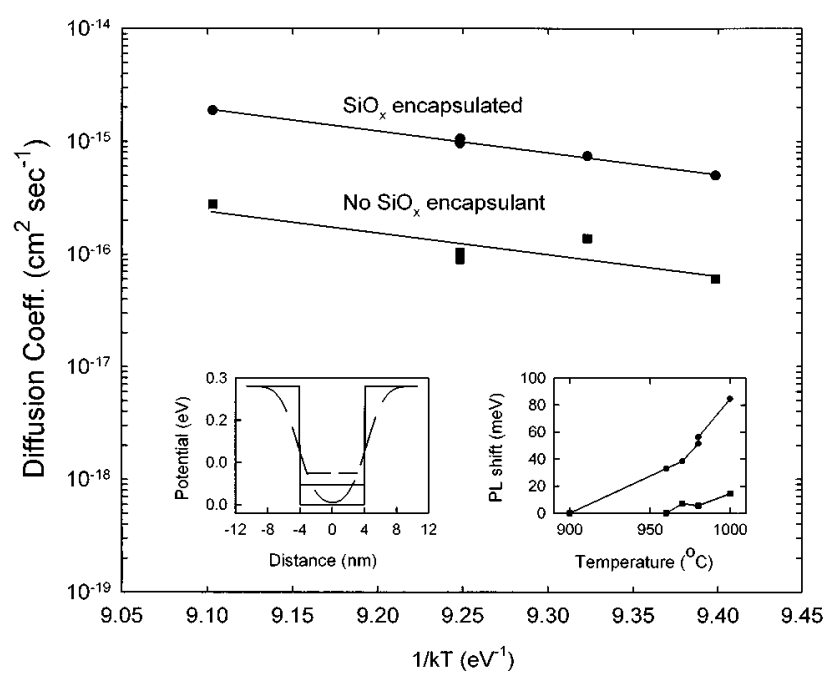

FIG. 2. A plot of the deduced values of the diffusion coefficient vs $1 / \mathrm{kT}$ for both the capped and the uncapped samples. The left inset shows the conduction band potential profile of an as-grown and an annealed sample at $980{ }^{\circ} \mathrm{C}$ for $20 \mathrm{~s}$. The right inset shows the measured PL shift as temperature is changed.

the electron and the hh were considered to be 0.067 and 0.48 $m$, respectively (where $m$ is the free electron mass). The band gap energy as a function of the $\mathrm{Al}$ composition, $x(z)$, was approximated by

$$
E_{g}(\mathrm{eV})=1.43+1.44 x(z)
$$

and the energy offset ratio of the conduction to the valence band was taken to be $65: 35$. The $n=1 e$-hh transition wavelength for the $80 \AA \mathrm{QW}$ was calculated to be $834.6 \mathrm{~nm}$. This value agrees with the measured PL of the structure which was found to peak at $\lambda=835 \mathrm{~nm}$. The values of the diffusion coefficient that correspond to the blue shift at the annealing temperatures were calculated by an iterative procedure. For an assumed value of $D$, the $e 1$-hhl transition energy of the intermixed $\mathrm{QW}$ was calculated using the transmission matrix method $^{16}$ and compared to the measured value until they were less than $0.5 \mathrm{meV}$ apart. In Fig. 2, one of the insets shows the potential profile of the conduction band after the sample has been annealed for $20 \mathrm{~s}$ at $980{ }^{\circ} \mathrm{C}$ assuming $D=8 \times 10^{-16} \mathrm{~cm}^{7} \mathrm{~s}^{-1}$, and the other inset shows the measured PL shift as the temperature is increased. Also in Fig. 2 we show the deduced values of $D$ plotted as a function of $(1 / k T)$ for both the capped and the uncapped samples. A curve fit of the data gives a single activation energy of $E_{a} \approx 4.4 \mathrm{eV}$. This value is in good agreement with the results reported in the literature. ${ }^{12,15}$ The fact that almost identical activation energies were obtained for both curves implies that the dissimilarity in disordering behavior between the $\mathrm{SiO}_{x}$ capped and uncapped sample is due to the difference in the number of vacancies generated at the surface of the samples. From Fig. 2, it is evident that the diffusion coefficient for the $\mathrm{SiO}_{x}$ capped sample is one order of magnitude greater than that for the uncapped samples.

A single-mode ridge waveguide was fabricated from an intermixed sample and the waveguiding losses were mea-

J. Appl. Phys., Vol. 80, No. 6, 15 September 1996 sured using a thermally scanned Fabry-Perot transmission technique. For this measurement, a cw Ti:sapphire laser with an intracavity frequency narrowing étalon was employed for stable narrow linewidth operation. The measurements of the Fabry-Perot finges indicated that scattering losses due to the vacancy induced disordering was around $10 \mathrm{~dB} / \mathrm{cm}$. While this value is still too high for the technique to be applicable to the integration of several devices, a simple demonstration of its intended use has been performed with the integrated all-optical devices described in the next section.

\section{APPLICATIONS TO ALL-OPTICAL DEVICES}

The first device described in this article is the zero-gap nonlinear directional coupler (NLDC) which consists of a single mode input waveguide, a dual-mode coupling section, and two single-mode output waveguides as shown in Fig. 3(a). Except for the mode-beating section, all regions of the MQWs were intermixed to blue shift the band edge away from the device operating wavelength. After the silicon oxide was spun on the surface, the film was cured as described above. Then using a contact mask photolithographic process to define windows measuring $100 \times 500(\mu \mathrm{m})^{2}$ in positive photoresist, a buffered oxide etch BOE was used to remove the oxide film within the exposed windows. After removing the photoresist, the sample was subjected to rapid thermal annealing at $980{ }^{\circ} \mathrm{C}$ for $20 \mathrm{~s}$ to diffuse the vacancies into the MQW region. Thus the whole sample except for the windowed sections, was intermixed. The zero-gap directional coupler was defined using a second step photolithography and aligned carefully so that the double-mode waveguide sections are exactly within the nonintermixed windows. Using a wet chemical etch consisting of $\mathrm{H}_{3} \mathrm{PO}_{4}: \mathrm{H}_{2} \mathrm{O}_{2}: \mathrm{H}_{2} \mathrm{O}$ solution in the ratios of $1: 1: 10$, a $350 \mathrm{~nm}$ thickness of the cladding layer is etched off to form cladding ridges for 3.4$\mu \mathrm{m}$-wide double-mode switching sections with $1.7-\mu \mathrm{m}$-wide single mode waveguides. Finally the GaAs substrate was polished down to $100 \mu \mathrm{m}$ thickness and the sample was cleaved to a total length of $1.7 \mathrm{~mm}$ assuring good facets.

The second device is the integrated Mach-Zehnder (MZ) optical modulator shown in Fig. 3(b). It contains a $500-\mu \mathrm{m}$-long active switching region of nonintermixed MQW in one arm, while the other arm and the rest of the structure were made of disordered MQW. The same process described for the above was also used in the fabrication of this device except that in this case the defined windows measured $20 \times 500(\mu \mathrm{m})^{2}$. PL measurements showed a distinctive difference between the peak wavelengths of the luminescence from the windowed (nonintermixed) and the intermixed regions. This indicates that the technique is highly area selective.

To characterize the switching devices we used a pumpprobe setup with a mode-locked Ti-sapphire laser as the light source. The laser wavelength was tunable in the range from 800 to $900 \mathrm{~nm}$ with a pulse width of $150 \mathrm{fs}$. In this pumpprobe experiment, the laser beam was split into a strong pump beam and a weaker probe beam that was modulated into rectangular pulses using a mechanical chopper. A halfwave plate was used to rotate the polarization of the pump beam so that it was polarized in the horizontal direction, 

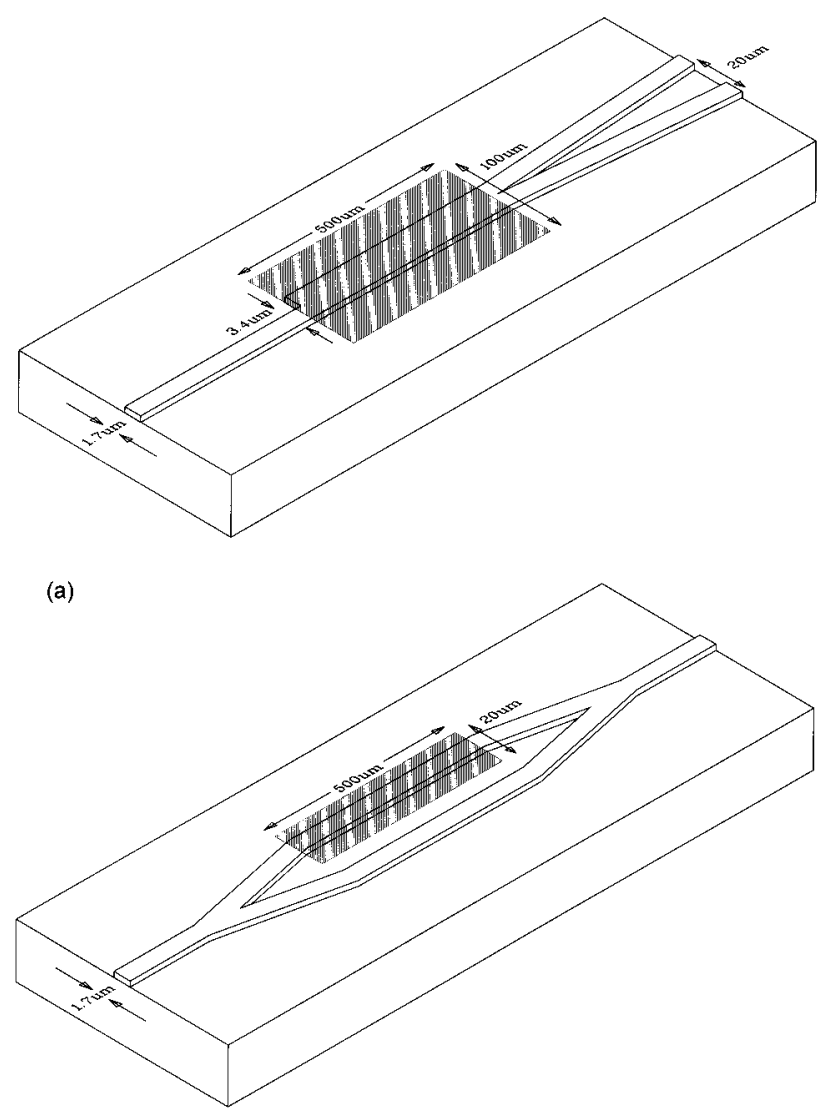

(b)

FIG. 3. Schematics of integrated devices. (a) Nonlinear coupler switch (b) Symmetric nonlinear Mach-Zehnder modulator. The shaded regions represent the nonintermixed areas, all other areas are intermixed.

while the probe beam was polarized in the vertical direction. The temporal delay between the two pulses was adjusted by changing the path length traversed by the probe beam. This was accomplished using a retroreflector mounted on a

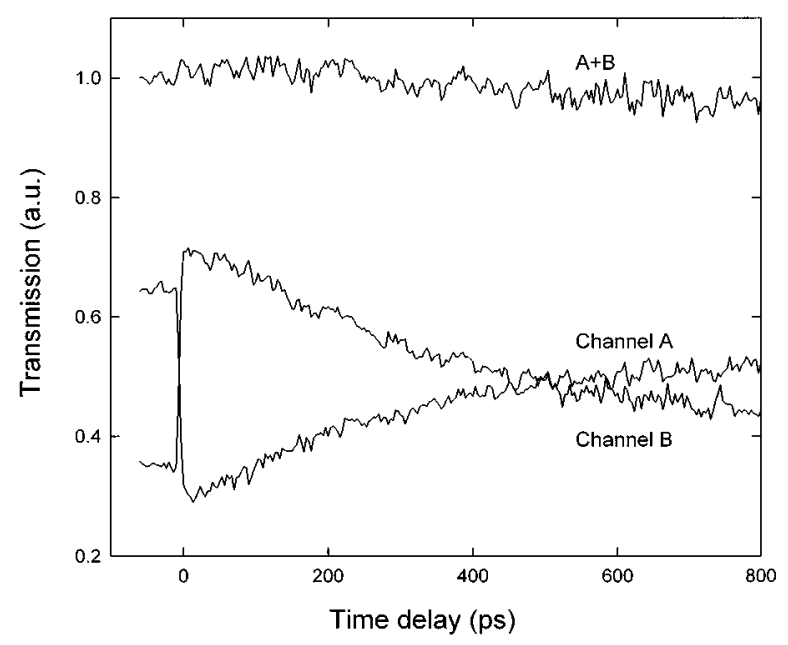

FIG. 4. Switching of the transmission of the probe pulses through the output channels A and B as a function of the time delay with the pump pulses.

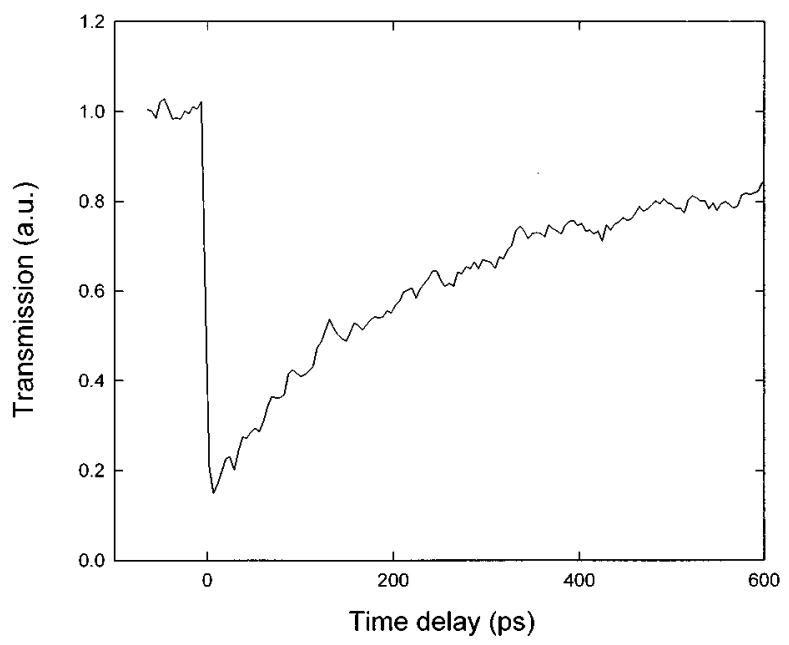

FIG. 5. Probe transmission in an integrated at Mach-Zehnder interferometer as a function of the delay between the probe and the pump pulses.

computer-controlled delay stage. The two beams were recombined colinearly and focused into the input waveguide using a $\times 45$ microscope objective lens with a numerical aperture of 0.65 , resulting in an estimated coupling efficiency of $10 \%$. To observe the output beam on a video monitor, another lens was used to image the output ends of the waveguide onto a CCD camera. The pump beam was filtered out by the use of a cross-polarizer placed at the output of the device. A Si photodetector connected to a lock-in amplifier was used to detect the mechanically chopped probe beam from either output port of the device. Figure 4 shows the transmission of the probe signal in both output ports of the NLDC as a function of the delay between the pump and the probe beams. The initial 63:37 split ratio of the output ports was switched to 30:70 with an exponential recovery time constant of around $300 \mathrm{ps}$. The total power through the two output ports remained constant and hence indicated that negligible nonlinear absorption took place.

The same optical setup was used to measure the switching performance of the integrated Mach-Zehnder device. Figure 5 shows the output power being modulated by the injection of a strong optical pump pulse. The device has a switch contrast of 7 to 1 and it too exhibited an exponential recovery time constant of about $300 \mathrm{ps}$.

\section{CONCLUSIONS}

We have developed an inexpensive and reliable process for the area-selective disordering of MQW structures. The scattering losses due to the vacancy induced disordering was measured to be less than $10 \mathrm{~dB} / \mathrm{cm}$ and work is currently underway to further improve on the waveguiding quality of the disordered MQWs. This technique has been applied to the fabrication of two integrated optical devices. In both devices, the mechanism for the switching is the nonlinear refractive index that is caused by photo-generated carriers. Since this mechanism entails absorption of some of the pump beam, it is hence very important that the optical absorption be confined to the active sections only. Selective area disor- 
dering is shown to be very effective at defining regions of different band gap energies. Hence it can be ensured that the energy of the pump laser beam is too low in comparison to the band gap energy of the passive regions to be absorbed and the free carriers are only created in the nonintermixed active sections. The controlled selective area intermixing of MQW structures will potentially play a significant role in the advancement of photonic integrated circuits.

${ }^{1}$ J. H. Marsh, Semicond. Sci. Technol. 8, 1136 (1993).

${ }^{2}$ M. Aoki, H. Sano, M. Suzuki, M. Takahashi, K. Uomi, and A. Takay, Electron. Lett. 27, 2138 (1991).

${ }^{3}$ D. Kirillov, J. L. Merz, P. D. Dapkus, and J. J. Coleman, J. Appl. Phys. 55, 1150 (1984).

${ }^{4}$ J. E. Epler, R. D. Burnham, R. L. Thornton, T. L. Paoli, and M. C. Bashaw, Appl. Phys. Lett. 49, 1447 (1986).

${ }^{5}$ Y. Hirayama, Y. Suzuki, and H. Okamoto, Jpn. J. Appl. Phys. 24, 1498 (1985).
${ }^{6}$ J. Cibert, P. M. Petroff, D. J. Werder, S. J. Pearton, A. C. Gossard, and E. English, Appl. Phys. Lett. 49, 223 (1986).

${ }^{7}$ W. D. Laidig, N. Holonyak, Jr., M. D. Camras, K. Hess, J. J. Coleman, P. D. Dapkus, and J. Berdeen, Appl. Phys. Lett. 38, 776 (1981).

${ }^{8}$ D. G. Deppe, L. J. Guido, N. Holonyak, Jr., K. C. Hsieh, R. D. Burnham, R. L. Thornton, and T. L. Paoli, Appl. Phys. Lett. 49, 510 (1986).

${ }^{9}$ E. S. Koteles, B. Elman, R. P. Holmstron, P. Melman, J. Y. Chi, Xin Wen, J. Powers, and D. Owens, Superlattices Microstruct. 5, 321 (1989).

${ }^{10}$ E. S. Koteles, B. Elma, P. Melman, J. Y. Chi, and C. A. Armento, Opt. Quantum Electron. 23, S779 (1991).

${ }^{11}$ M. Ghisoni, P. J. Stevens, G. Parry, and J. S. Roberts, Opt. Quartum Electron. 23, S915 (1991).

${ }^{12}$ I. Gontijo, T. Kraus, J. H. Marsh, and R. M. De La Rue, J. Quantum Electron. 30, 1189 (1994), and references therein.

${ }^{13}$ S. Shi, P. Li Kam Wa, A. Miller, J. Pamulapati, P. Cooke, and Mitra Dutta, Appl. Phys. Lett. 66, 79 (1995).

${ }^{14}$ T. E. Schlesinger and T. Kuech, Appl. Phys. Lett. 49, 519 (1986).

${ }^{15}$ J. D. Ralston, S. O'Brien, G. W. Wicks, and L. F. Eastman, Appl. Phys. Lett. 52, 1511 (1988).

${ }^{16}$ Y. Zebda and A. M. Kan'an, J. Appl. Phys. 72, 559 (1992). 